Service social

\title{
Programme de soutien aux enfants et aux adolescents endeuillés. Intervention de groupe destinée aux enfants, aux adolescents et à leurs parents
}

\section{Agathe Bisson}

Volume 45, numéro 3, 1996

Santé

URI : https://id.erudit.org/iderudit/706740ar

DOI : https://doi.org/10.7202/706740ar

Aller au sommaire du numéro

Éditeur(s)

École de service social de l'Université Laval

ISSN

1708-1734 (numérique)

Découvrir la revue

Citer cet article

Bisson, A. (1996). Programme de soutien aux enfants et aux adolescents endeuillés. Intervention de groupe destinée aux enfants, aux adolescents et à leurs parents. Service social, 45(3), 103-122. https://doi.org/10.7202/706740ar
Résumé de l'article

Le Programme de soutien aux enfants et aux adolescents endeuillés est un programme d'intervention psychopédagogique de groupe s'adressant aux enfants de 5 à 18 ans qui ont perdu un être cher. Le programme a été mis sur pied par un groupe de travailleurs sociaux de l'Hôpital général Saint-Boniface à Winnipeg, dans le but d'aider les enfants à surmonter les problèmes sociaux et psychologiques auxquels ils font face à la suite du décès. L'auteure fournit une description du programme, des thèmes et des questions soulevés en groupe et de la manière dont on a répondu à diverses situations. 


\section{Programme de soutien aux enfants et aux adolescents endeuillés}

\section{Intervention de groupe destinée aux enfants, aux adolescents et à leurs parents}

Agathe BISSON

Travailleuse sociale Hôpital général Saint-Boniface, Winnipeg

\section{INTRODUCTION}

Travailleurs sociaux dans un grand hôpital d'enseignement et de soins tertiaires, nous avons souvent l'occasion de répondre au personnel, aux patients et aux familles qui nous consultent sur la manière d'informer les enfants de la mort imminente d'un parent ou d'un proche et sur la façon de les aider à vivre leur deuil après le décès. $\mathrm{D}^{\prime}$ autant plus que nous avons prêté notre concours au programme de groupe "Kids Can Cope», destiné aux enfants et aux adolescents dont un proche est atteint du cancer (Taylor, Acheson et Farber, 1993). Parmi les enfants qui y sont inscrits, certains ont un proche gravement malade ou mourant dont la mort survient pendant la durée du programme 
ou peu après. Le manque de groupes de soutien pour les enfants en deuil dans notre localité a entraîné la création du Programme de soutien aux enfants et aux adolescents endeuillés de l'Hôpital général Saint-Boniface. Nous avons consacré un certain temps à examiner la documentation pertinente et à consulter les coordonnateurs des programmes existants, mis sur pied aux États-Unis pour la plupart. Notre programme s'inspire largement du «St. Mary's Young Person's Grief Support Program» de Duluth au Minnesota. Nous tenons à remercier M. Ben Wolfe, directeur du St. Mary's Grief Support Center, de sa précieuse collaboration pendant la planification du programme.

Certains auteurs comparent le deuil à un orage qu'on ne voyait pas venir et qui nous prend souvent par surprise. La pluie qui l'accompagne peut tomber tout doucement ou encore subitement, avec le ciel qui s'assombrit, les éclairs et le tonnerre. Cette pluie est parfois torrentielle, emportant tout sur son passage, puis elle laisse place à un ciel moins gris, à une éclaircie, à un arc-en-ciel (Ending and Beginnings, National Childhood Grief Institute, 1991). On a aussi comparé le deuil à l'océan, à des vagues de douleur et de confusion qui vont et viennent (Heegaard, 1988).

On peut lire dans de nombreux ouvrages que le deuil vécu par les enfants est différent de celui des adultes (Doka, 1995 ; Webb, 1993; Grollman, 1976; Krementz, 1981; Baker et al., 1992; Silverman et Worden, 1992; Raphael, 1983; Betz et Poster, 1984). Les enfants et les adolescents qui viennent de perdre un être cher traversent l'un des moments les plus durs et éprouvants qui soient. C'est pendant l'enfance que nous posons les jalons de notre croissance et de notre développement. Pendant l'adolescence, nous cherchons à nous définir comme individu et à acquérir plus d'autonomie par rapport à notre famille. Le deuil vient perturber les tâches développementales de l'enfant et la vie familiale sous tous ses aspects. Les enfants perdent soudainement le sentiment d'invulnérabilité qui les protégeait du chaos et des incertitudes du monde des adultes. Ils ne retrouvent plus la structure et la stabilité qu'il leur faut.

L'âge cognitif de l'enfant, son vécu par rapport à la mort, sa personnalité, sa relation avec le défunt, sa compréhension de la cause du décès et le type de communication dans la famille sont autant de facteurs qui influent sur sa compréhension de la mort. 
Les signes de deuil chez l'enfant peuvent être moins évidents que chez les adultes. Si certains enfants expriment leurs émotions spontanément et ouvertement, d'autres trouvent l'exercice difficile et même terrifiant, surtout s'ils grandissent dans un milieu où l'on ne s'exprime pas, où les sentiments sont gardés pour soi. Les enfants expriment souvent leur désespoir, leur tristesse, leur rage, leur confusion et leur peur d'une manière qui n'est pas considérée comme une réaction à un deuil. Les émotions ressenties peuvent prendre diverses formes (explosions d'injures ou réactions physiques, retrait social, difficultés scolaires, plaintes de douleurs physiques, troubles du sommeil ou de l'alimentation, comportement régressif, cauchemars ou attitude de l'enfant très sage, qui a décidé de soutenir le parent en deuil). L'expression de leur deuil passe souvent inaperçue aux yeux des adultes (Wolfe, 1987).

La mort fait partie de la vie. Elle est universelle et inévitable; aucun adulte ou enfant n'échappe à cette réalité. Or, parler de la mort met souvent mal à l'aise. Si la pensée même de la mort dérange les adultes, l'enfant sentira leur angoisse et leur tension. "Vivre un deuil est un processus, s'en remettre est un choix. Nous ne pouvons expliquer la mort aux enfants qu'après l'avoir démythifiée nous-mêmes » (Grollman, 1976). Les enfants ont besoin qu'on les aide et les encourage à comprendre le deuil et à exprimer leurs émotions, afin qu'ils puissent s'en remettre. Pour bien vivre un deuil, il est essentiel de communiquer et de s'exprimer. La communication engendre l'espoir. En entourant la mort de silence et de mystère, on prive les enfants d'une occasion importante de vivre et de partager leur deuil et ce, peu importe leur âge (Grollman, 1976; Doka, 1995). Ce n'est pas en refusant d'accepter une tragédie que l'on fait preuve d'une bonne santé mentale, mais plutôt en reconnaissant franchement la douleur de la séparation. Devant la mort d'un être cher, les enfants ont besoin de soutien, de compréhension, d'information, de propos rassurants, d'amour et de patience. Montrer son affection et son intérêt est le plus beau cadeau que l'on puisse faire à un enfant en deuil. Grollman (1976) souligne qu'un enfant ayant subi une perte a besoin tout autant de compassion que de compréhension.

Si l'on n'est pas encouragé ou autorisé à exprimer ses sentiments, le deuil sera refoulé et se manifestera des années plus tard sous forme de comportements autodestructeurs, d'abus d'intoxiquants, de dévalorisation ou de dépression (Heinzer, 
1995). Selon Bertoia et Allan (1988), la psychopathologie de l'adulte est beaucoup plus courante parmi les personnes qui ont nié le décès d'un parent ou qui n'ont pas surmonté ce deuil pendant leur enfance.

L'importance d'aider les enfants endeuillés est bien documentée. Nous savons également que les enfants peuvent rarement compter sur les adultes de la famille, eux-mêmes dépassés par le deuil et les changements familiaux, et qu'ils détestent en général être différents de leurs camarades et pointés du doigt, même si un décès est survenu dans leur famille (Krementz, 1981). Ces facteurs tendent à justifier la formation de groupes. En se fondant sur leurs expériences respectives par rapport à un point qu'ils ont en commun, les enfants sont en mesure de se soutenir les uns les autres.

\section{DESCRIPTION DU PROGRAMME}

Cet article décrit un programme d'intervention psychopédagogique de groupe appelé Programme de soutien aux enfants et aux adolescents endeuillés, qui s'adresse aux jeunes qui ont perdu un être cher à la suite d'un décès. Le programme vise à aider les enfants à surmonter les problèmes sociaux et psychologiques auxquels ils font face à la suite de cette perte.

Créé en novembre 1989, le programme est proposé deux fois par année, au printemps et à l'automne. Il vise les objectifs suivants :

1. réduire le sentiment d'isolement en donnant aux enfants l'occasion de rencontrer d'autres enfants qui ont vécu une perte semblable à la leur ;

2. éduquer les enfants sur le deuil dans un climat favorable où ils se sentiront soutenus ;

3. encourager les enfants à exprimer leur pensée et leurs sentiments par rapport à la mort et à considérer cette démarche comme normale ;

4. donner l'occasion aux enfants d'augmenter leur répertoire de comportements d'adaptation en leur proposant un éventail d'attitudes, de styles et de stratégies;

5. aborder la question du deuil dans une optique familiale;

6. apprendre de l'expérience de ces enfants, afin de mieux orienter les recherches à venir et l'élaboration de programmes. 
À la demande des parents, ceux-ci se réunissent simultanément depuis 1992. Le groupe de parents vise deux objectifs:

1. conseiller et orienter les parents afin de les aider à soutenir leurs enfants en deuil ;

2. fournir aux parents tout le soutien nécessaire pour surmonter leur propre deuil et s'adapter aux changements survenus dans leur famille.

Le deuil n'épargnant aucun membre de la famille, l'ajout d'un groupe de parents constituait une évolution naturelle. Le programme vise principalement à encourager la communication et à maintenir un lien de confiance entre les parents et les enfants. Il fournit aussi l'occasion de rassurer et de soutenir les parents. On a déjà écrit que le parent qui s'adapte bien à un deuil permet à son enfant de pleurer la perte et de s'en remettre dans des conditions saines et favorables (Gray, 1989).

Le programme s'adresse aux enfants et aux adolescents de 5 à 18 ans et à leurs parents qui ont perdu un être cher. Les participants sont dirigés vers ce programme par le personnel hospitalier, des médecins de famille, des organismes communautaires et des écoles. Bon nombre se présentent spontanément. Le nombre d'enfants inscrits varie entre 16 et 25 . La participation des parents est encouragée mais pas obligatoire. Le programme n'est annoncé que dans le bulletin hebdomadaire de l'Hôpital et la priorité est donnée aux familles dont un proche est décédé à l'Hôpital général Saint-Boniface. Le temps écoulé depuis le décès varie entre deux mois et trois ans. Dans la majorité des cas, le décès est survenu dans la dernière année. La plupart des familles viennent de Winnipeg ou vivent à l'intérieur d'un périmètre de $100 \mathrm{~km}$ de cette ville.

Le tableau 1 (page suivante) donne le nombre d'enfants inscrits, indique qui est décédé et mentionne la cause du décès, de 1989 à 1996.

Une fois les inscriptions effectuées, les animateurs communiquent avec les parents, leur expliquent le programme et conviennent d'une visite à domicile afin de rencontrer la famille. Cette visite est cruciale. Elle donne l'occasion à l'animateur de rencontrer les enfants, d'entrer en relation avec eux et de répondre aux questions qu'ils pourraient avoir au sujet du programme. Elle lui permet aussi d'évaluer la situation de la famille et sa capacité d'adaptation. L'information ainsi recueillie constitue une présélection dont il sera question plus loin. Les enfants sont répartis en fonction de leur âge et de leur degré de maturité dans 
TABLEAU 1

Programme de soutien aux enfants et aux adolescents endeuillés Novembre 1989 à novembre 1996 - 14 groupes

\begin{tabular}{lcc}
\hline $\begin{array}{c}\text { Relation avec } \\
\text { la personne décédée }\end{array}$ & $\begin{array}{c}\text { Nombre total } \\
\text { d'enfants }\end{array}$ & Pourcentage \\
\hline Mère & 61 \\
Père & 135 & \\
Frère, soeur & 36 & 75,1 \\
Grand-parent & 15 & 13,8 \\
Autre personne & 14 & 5,7 \\
Total & $\mathbf{2 6 1}$ & 5,4 \\
& & $\mathbf{1 0 0 , 0} \%$ \\
\hline Cause du décès & Nombre total & Pourcentage \\
& d'enfants & \\
\hline Cancer & 104 & 39,8 \\
Autres maladies & 62 & 23,8 \\
Accident & 53 & 20,3 \\
Suicide & 29 & 11,1 \\
Autre cause & 13 & 5,0 \\
Total & $\mathbf{2 6 1}$ & $\mathbf{1 0 0 , 0} \%$ \\
\hline
\end{tabular}

trois ou quatre groupes formés de quatre à huit membres. Chaque groupe est animé par deux travailleurs sociaux (des psychologues et des étudiants en service social occupent parfois ce rôle).

Le programme comprend sept (huit dans certains cas) séances hebdomadaires tenues en dehors des heures de classe. Les parents, les enfants et les animateurs commencent par se rassembler dans une grande salle polyvalente pour prendre un goûter ${ }^{1}$, puis se divisent en petits groupes. La huitième séance ne s'adresse qu'aux parents et aux animateurs. Elle donne l'occasion aux parents de dire comment vont leurs enfants et aux animateurs de décrire le déroulement des séances antérieures, tout en respectant le droit à la vie privée des enfants.

1. Nous tenons à remercier l'Auxiliaire de l'Hôpital, qui fournit tous les ans des fonds servant à payer la nourriture et le matériel nécessaire aux activités de groupe, ainsi que le département de bénévolat, qui offre les services d'un bénévole pour la coordination des goûters. 
L'objectif visé par la première séance est de permettre aux enfants de faire connaissance et d'établir un climat de confiance et une cohésion à l'intérieur du groupe. Les enfants sont invités à raconter leur histoire (qui est décédé, comment, où...), rendant ainsi la discussion sur la mort socialement acceptable et approuvée. On explore les conceptions et les réactions de chaque enfant face à la mort. Le simple fait de dire le mot "mort» entraîne souvent de vives émotions et des craintes. Voici un bon exemple de recours au symbolisme à cet égard par un petit garçon de huit ans. David savait bien que son père était gravement malade et que sa mort était imminente. Sa mère lui avait demandé comment il voulait qu'elle lui apprenne la nouvelle. «Tu me diras que papa tond le gazon. » Revenant un jour de l'hôpital elle dit à David: "Ton père tond le gazon. » David s'est retourné vers la fenêtre, a regardé la neige et a dit: "Je sais qu'il ne tond pas le gazon, il est mort!» (voir les dessins des enfants représentant la mort, figures 1 et 2 ).

La deuxième séance est consacrée aux souvenirs se rapportant au défunt. C'est avec enthousiasme que les enfants font partager les trésors (des souvenirs ou des objets personnels qu'ils apportent au groupe) qui les lient au défunt. On y trouve

Figure 1

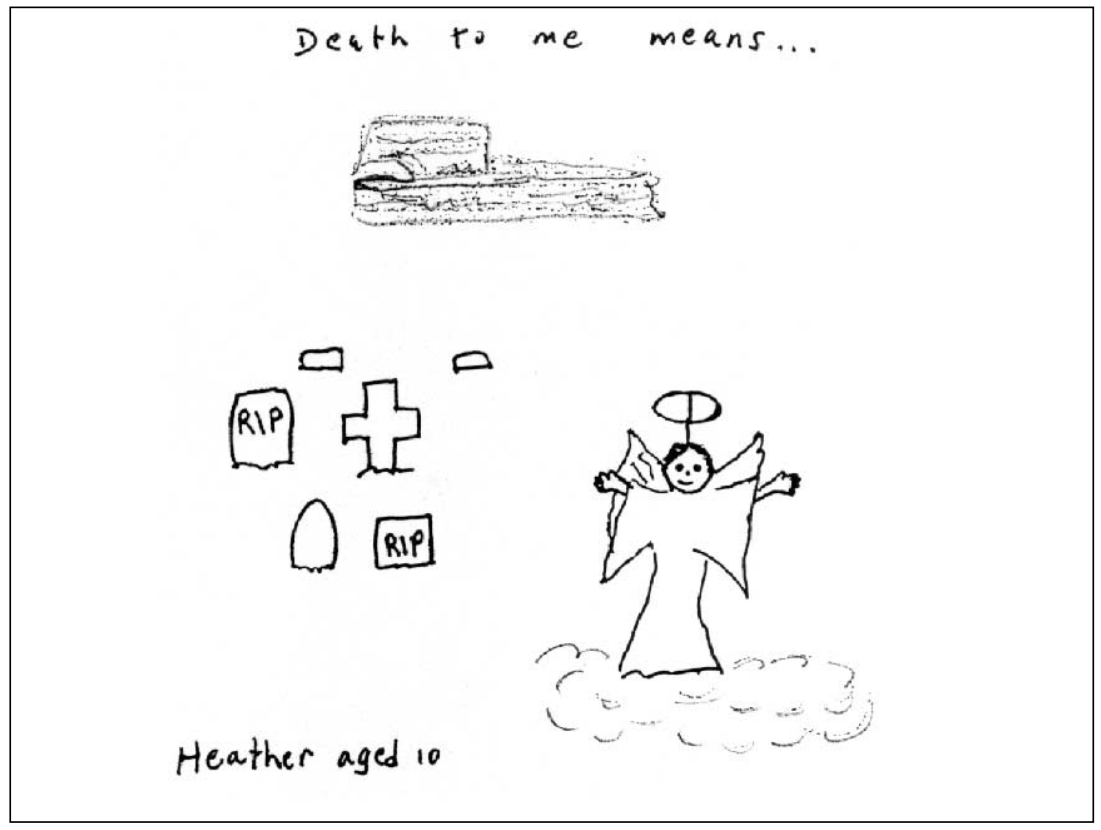


Figure 2

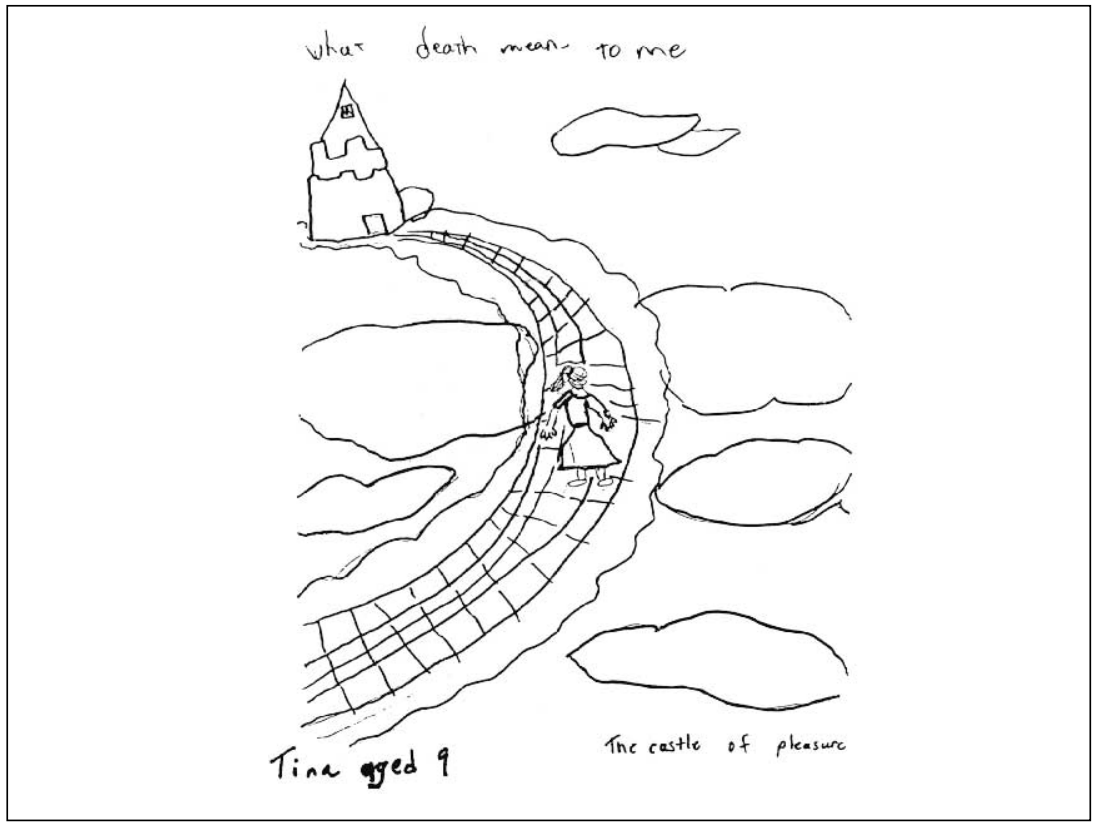

des photos encadrées du défunt, des albums de photos, des vêtements, des bijoux et des jouets offerts par le défunt. Par exemple, une fillette de neuf ans a apporté le contenant de sel à l'ail que son père utilisait à chaque repas. En faisant partager leurs souvenirs, les enfants parlent de leurs activités avec le défunt. Les animateurs font remarquer que certains souvenirs sont bons, tandis que d'autres le sont moins. Les enfants semblent accorder beaucoup d'importance aux souvenirs et à la commémoration.

La troisième séance aborde la question des funérailles et des rituels associés à la mort. Elle comprend une discussion sur les faits et les mythes entourant la mort, notamment sur des sujets que nous ne connaissons ou ne comprenons pas. Les enfants parlent souvent de leur croyance par rapport à la «vie après la mort » ainsi que de l'âme ou de l'esprit du défunt. La figure 3 représente le dessin de Kelly illustrant les funérailles de sa maman.

La quatrième séance est consacrée aux sentiments liés au décès d'un proche. Les enfants sont invités à nommer et à reconnaître un éventail de sentiments d'intensité variable 
Figure 3

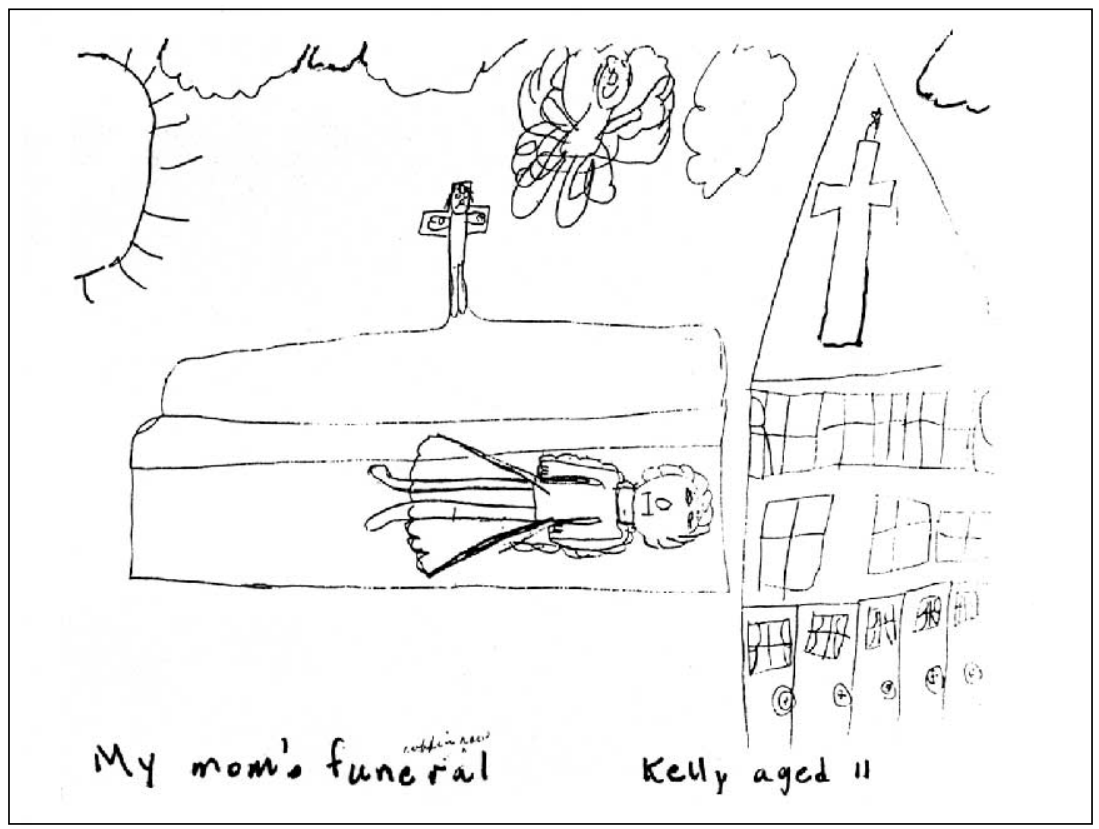

Figure 4

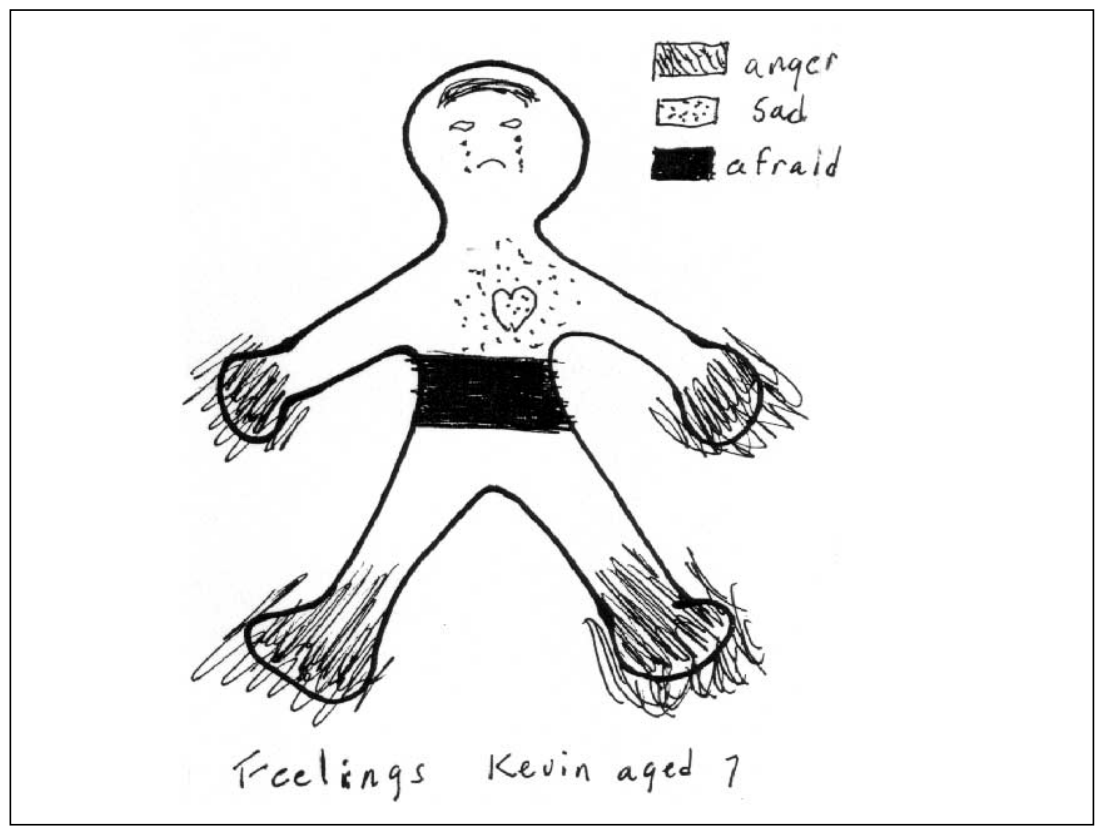


(tristesse, rage, confusion, regrets, culpabilité), qui sont ensuite considérés comme normaux. La figure 4 montre certains sentiments que l'enfant ressent dans son corps.

La cinquième séance porte sur la capacité d'adaptation. Elle vise à augmenter le répertoire de stratégies à adopter et à faire réaliser aux enfants que certaines réponses d'adaptation sont plus efficaces que d'autres. Les enfants sont encouragés à exprimer des sentiments comme la colère, en tant que moyen d'évacuer une partie de leur douleur et de leur peine sans pour autant nuire à eux-mêmes, aux autres et aux biens matériels. On les encourage aussi à définir leur réseau d'entraide, c'est-à-dire à nommer les personnes sur qui ils peuvent ou pourraient compter.

La sixième séance s'attarde sur les changements produits sur le plan personnel et familial. Les enfants peuvent être invités à faire trois dessins illustrant ce que les membres de leur famille faisaient dans le passé, ce qu'ils font aujourd'hui et ce qu'ils feront demain. L'enfant est ainsi encouragé à dire ce qu'il ressent par rapport aux autres membres de la famille, à la dynamique familiale et aux conséquences du décès dans la vie familiale. Le recours au dessin permet aux enfants qui ont de la difficulté à verbaliser leur pensée et leurs sentiments de s'exprimer sans se sentir menacés. Il peut aussi aider les enfants à prendre davantage conscience des sentiments qu'ils éprouvent.

Les animateurs encouragent les enfants à parler de leurs dessins. Les animateurs tiennent parfois compte des dessins, mais ils ne les analysent jamais. L'exercice amène également l'enfant à réfléchir sur ses attentes par rapport à lui-même et à sa famille. Les enfants ont besoin qu'on leur donne la permission de «continuer». Il faut toutefois préciser que «continuer» n'est pas la même chose qu' «oublier». Les enfants ont besoin de se rappeler (Wolfe, 1987). La dernière séance est consacrée à l'évaluation et à la conclusion du programme. Chaque participant reçoit un certificat de participation et le tout se termine par une dégustation de pizza.

Pendant chacune des séances, on organise des activités propres à chaque niveau de développement, afin de mieux répondre aux objectifs de la séance. Par exemple, les jeunes enfants peuvent être invités à fabriquer des marionnettes en sacs de papier représentant divers sentiments qu'ils éprouvent face à la mort et à s'en servir pour exprimer ces sentiments. Les dessins, les collages et d'autres moyens d'expression du genre sont souvent utilisés avec les plus jeunes, alors que les adolescents 
ont généralement moins de difficultés à exprimer leurs pensées et leurs sentiments verbalement ou par écrit (biographie du défunt, poème d'adieu, etc.).

Après chaque séance, les animateurs se rencontrent pour faire leur compte rendu et se consulter. Deux ou trois enfants d'une même famille sont parfois inscrits dans des groupes différents et leur parent fait aussi partie du groupe de parents. L'échange d'information permet aux animateurs de mieux comprendre la façon dont un enfant en particulier réagit et s'adapte au décès.

\section{THÈMES QUI REVIENNENT SOUVENT}

Au cours des sept ans d'existence du programme, on a pu dégager les trois thèmes suivants: 1. l'universalité et la légitimité, 2. la méconnaissance de la mort et 3 . les thèmes propres à chaque groupe d'âge.

\section{Universalité, légitimité}

Le fait de réunir des enfants vivant une situation similaire a une valeur hautement thérapeutique. Nous savons que les enfants et les adolescents n'aiment pas être considérés comme différents par leurs camarades et qu'ils évitent généralement de parler du décès à leurs amis. À l'intérieur du groupe, ils ont l'impression d'être compris et soutenus moralement.

Plus les mois passaient, moins les gens voulaient écouter. Personne n'aime entendre des pensées morbides et tous évitaient le sujet. Une fois par semaine, je pouvais partager mes sentiments avec d'autres personnes qui m'écoutaient et me comprenaient. Personne ne me disait de me taire ou n'essayait de changer de sujet. Ça fait du bien de savoir que je peux aller à un endroit où je rencontre des gens à qui je peux parler. J'ai remarqué que personne n'avait peur de dire le mot «mort» ou «quand ton père est mort». On m'a permis de discuter de ces choses ouvertement et librement. C'était peut-être ce qu'il me fallait pour commencer à me remettre de ma peine (Carla, 14 ans).

Ça m'a aidé beaucoup, probablement parce que je n'avais jamais donné autant de détails sur tous les faits ou sur tout ce qui s'était passé au mois d'avril (quand mon père est mort) (Kyle, 16 ans). 
Rencontrer d'autres enfants crée un sentiment d'appartenance et de reconnaissance de la nature universelle et légitime de leurs sentiments. "Mark (qui a 16 ans et qui a lui aussi perdu son père) et moi sommes devenus amis très rapidement » (Jason, 16 ans). Les liens ainsi créés permettent aux enfants de s'entraider et d'apprendre de l'expérience des autres.

\section{Méconnaissance de la mort}

La mission éducative du programme est jugée importante. En règle générale, les enfants associent bien des craintes et des fabulations à la mort. Malheureusement, l'idée qu'on se fait de la mort est souvent inexacte ou le fruit de l'imagination. Par exemple, les enfants peuvent voir la mort sous les traits d'un monstre ou du bonhomme sept heures, ou encore penser qu'elle ne frappe que les gens méchants ou les personnes âgées. De plus, le silence et le secret entourant la mort ou l'utilisation d'euphémismes («elle nous a quittés, elle s'est éteinte, nous l'avons perdu...») ne font qu'augmenter la confusion et accroître les craintes et les fabulations des enfants.

Il n'est pas rare de rencontrer des enfants qui se sentent responsables du décès, c'est-à-dire qui croient que leurs pensées ou leurs gestes en sont la cause. Prenons l'exemple de Roberta, 8 ans, qui s'accusait toujours d'avoir causé la mort de son père. Lorsqu'on l'a interrogée à ce sujet, elle a raconté aux membres du groupe comment son père était tombé par terre et s'était blessé lorsqu'elle avait enlevé la chaise de cuisine sur laquelle il s'apprêtait à s'asseoir. Il était entré à l'hôpital quelques jours plus tard et est mort peu après d'une maladie du rein. La petite fille était convaincue que ses problèmes rénaux étaient attribuables à sa chute, alors qu'ils étaient dus à une consommation excessive de drogue durant une longue période (ce qu'elle ignorait). Les enfants peuvent aussi se culpabiliser ou exprimer des regrets par rapport à des choses qu'ils auraient dû faire ou dire quand la personne était vivante. Ce genre de réaction s'applique à toutes les causes de décès, mais est plus courant parmi les enfants dont le proche s'est suicidé et qui cherchent à comprendre comment la personne en est arrivée à faire un tel choix. Bien des enfants ont peur de perdre le parent qui leur reste, comme le petit Allan, 7 ans, qui était convaincu que son père allait mourir le mois suivant, parce qu'il allait avoir 48 ans (sa mère était morte d'une tumeur au cerveau à 48 ans). «Que m'arrivera-t-il? », se disent-ils. 
On devrait répondre aux questions des enfants sur la mort, les funérailles, les rituels et l'incinération honnêtement et correctement, en tenant compte de leur degré de compréhension. Il faut leur rappeler constamment les faits, c'est-à-dire que la mort n'a pas été causée par leurs pensées ou leurs actions. Les animateurs du groupe de parents encouragent ces derniers à faire de même, notamment en discutant de leur testament avec leurs enfants et en leur disant qui prendra soin d'eux et comment on s'organisera si le parent survivant venait à mourir. Cette façon de faire permet d'atténuer une partie de leurs craintes et de les rassurer.

Il est important d'essayer de découvrir ce qui se cache derrière les questions des enfants afin de pouvoir y répondre avec pertinence. Certains enfants font entrer Dieu en jeu dans la mort de leur proche. En disant que «Dieu l'a pris », l'enfant exprime peut-être des sentiments de crainte ou de colère envers Dieu.

\section{Thèmes propres à chaque groupe d'âge}

L'idée que les enfants se font de la mort varie en fonction de l'âge et du niveau de développement.

Les jeunes enfants ont non seulement une capacité d'attention limitée, mais leur «capacité de ressentir les choses » est tout aussi restreinte (Doka, 1995). Ils ne peuvent supporter longtemps la douleur affective, comme le démontre la réaction de Jannel, 5 ans, qui, après 15 minutes de discussion de groupe sur la mort de leur proche et ses funérailles, s'est écriée: "J'en ai assez de parler de la mort, ça me rend trop triste». L'animateur a alors décidé qu'il était temps de faire une pause et a proposé aux enfants de raconter des histoires drôles. La réaction a été immédiate et la salle s'est aussitôt emplie d'éclats de rire. Les adultes pourraient penser qu'ils n'ont pas de peine, qu'ils ont déjà oublié la perte, alors qu'en réalité on peut discerner leur peine dans les sentiments qu'ils expriment et les comportements qu'ils adoptent de façon intermittente longtemps après le décès. Nous avons constaté que les jeunes enfants ont besoin d'être mieux structurés et qu'ils doivent être engagés dans diverses activités tout en parlant. Ils prennent également les choses à la lettre et les réduisent à leur plus simple expression. Au beau milieu du discours d'une petite fille de 5 ans qui disait que son père avait été incinéré, un autre enfant du même âge a rétorqué: "Mais c'est impossible, un four est beaucoup trop petit pour une grande personne». Un autre enfant était d'avis que tous les 
membres de la famille devraient mourir en même temps, afin que personne ne soit triste. Ils ont également des idées bien arrêtées sur le ciel et l'enfer. Un enfant a déjà parlé des règles à respecter une fois qu'on est rendu au ciel.

Les enfants dans la période de latence (9 à 12 ans) commencent à reconnaître l'irrévocabilité et l'universalité de la mort. Ils ont tendance à s'attarder sur la façon dont la perte les affecte personnellement et à parler de leur vie sans cette personne. Ces enfants se demandent comment réagir aux taquineries à la cour de récréation et comment parvenir à faire leurs travaux, alors qu'ils sont dérangés par ce qui arrive à la maison. Par exemple, Jaimie, 10 ans, a parlé de la peine et de la douleur qu'elle a ressenties lorsqu'un garçon lui a crié dans le couloir de l'école deux jours après les funérailles: «Dis donc, qu'est-ce qui est arrivé à ton père? » (il s'était suicidé). Elle a raconté aux autres membres du groupe qu'elle avait voulu disparaître immédiatement, que le garçon connaissait probablement la cause du décès et qu'il voulait l'embêter. Les autres enfants ont été invités à parler d'expériences similaires et à trouver ensemble des stratégies à adopter lorsqu'elles se présentent. Les enfants ont fait preuve de beaucoup de créativité et d'entraide en tentant de contrer ces difficultés.

En règle générale, les adolescents voient d'un bon œil la possibilité de discuter de la perte qu'ils ont subie, surtout avec d'autres jeunes qui ont eux aussi perdu un être cher. Certains adolescents n'osent pas exprimer leurs sentiments à l'école et auprès de leurs amis afin de rester à la hauteur ou ils se sentent inutiles parce qu'ils n'arrivent pas à répondre aux attentes. Ils ont souvent moins de difficultés à exprimer leurs émotions à l'extérieur du cadre familial à une période de leur vie où ils cherchent à s'en démarquer. Ils peuvent également sentir qu'il n'y a personne dans la famille pour les soutenir, le parent survivant étant lui-même accablé par son propre deuil. Les adolescents assument souvent le rôle de parent auprès des plus jeunes tout en cherchant à soutenir le parent en deuil. Les participants font habituellement état de leur ressentiment et de leur colère à l'égard des responsabilités supplémentaires qu'ils assument à la maison depuis le décès et ils parlent du sentiment de culpabilité que ce ressentiment leur cause. La nouvelle situation familiale risque d'exacerber les tensions et les conflits habituels. Les adolescents attachent de la valeur et une grande signification au fait d'avoir la possibilité de dire ce qu'ils ressentent aux autres membres du groupe. 
Les membres d'une même famille ne vivent pas tous leur deuil en même temps. Le deuil des enfants s'étend sur une plus longue période, car ils vivent et revivent leur deuil en parvenant à chaque nouveau stade de leur développement et acquièrent en cours de route une meilleure compréhension de la mort. Tout dépend de l'âge: il peut se passer des semaines, des mois, voire des années avant que l'enfant réalise les conséquences que la mort d'une personne entraîne (Baker et al., 1992).

Extraits d'un poème à «mon père » :

Tu seras toujours à mes côtés

Dans mes yeux

Tu continueras de te révéler

Dans mon cour

Quand je me marierai

Jamais je n'oublierai

Tout ce que tu m'as donné

Tu fais partie de ma vie

Et maintenant que tu es parti

Je te dis je t'aime papa

Et je ne t'oublie pas

Michelle, 15 ans, $10^{\mathrm{e}}$ année

\section{Groupes de parents}

Parmi les thèmes qui reviennent souvent au sein des groupes de parents, mentionnons l'accablement et la colère par rapport au décès et à la modification des rôles qu'il entraîne, la solitude, les crises d'identité personnelle, les regrets, la culpabilité entourant le fait de répondre à ses propres besoins au détriment de ceux des enfants et la manière de surmonter son propre deuil. Les parents se demandent aussi jusqu'à quel point ils peuvent partager leur peine avec leurs enfants, comment les aider à s'adapter au deuil et quoi faire lorsque des personnes bien intentionnées mais qui ne comprennent pas donnent de mauvais conseils. 


\section{QUESTIONS RELATIVES AU PROGRAMME}

\section{Confidentialité du programme}

La confidentialité encourage les enfants à partager leur expérience personnelle et à exprimer leurs sentiments sans craintes. Elle favorise aussi la confiance et la cohésion au sein du groupe. Au départ, les jeunes enfants restaient si discrets qu'ils ne parlaient pas de leur expérience de groupe à leurs parents, ce qui nuisait à notre objectif qui est de favoriser la communication entre les enfants et leurs parents.

Nous encourageons maintenant les enfants à dire à leurs parents de quoi ils ont parlé dans le groupe et à décrire les activités auxquelles ils ont pris part. Nous les invitons à ne pas parler de l'expérience personnelle de leurs nouveaux amis afin de respecter leur intimité. Pendant la visite à domicile, nous expliquons aux parents et aux enfants que les animateurs ne rapporteront pas aux parents les propos des enfants à l'intérieur du groupe, sauf si ces propos les inquiètent vivement.

Les parents respectent en général le droit à la vie privée de leur enfant. Pour leur part, les enfants sont persuadés que les animateurs ne parleront pas derrière leur dos.

\section{Pression parentale en faveur de la participation}

La pression parentale est habituellement palpable pendant la visite à domicile, quand les enfants ne veulent pas se joindre au groupe ou se montrent ambivalents. L'animateur insiste sur le fait que la participation des enfants est strictement volontaire, qu'ils sont invités à assister aux premières séances et qu'ils ont le droit de mettre fin à leur participation en tout temps. Sur les 13 séries de séances regroupant en tout 261 enfants, seulement 10 ont abandonné le programme.

\section{Méthode de sélection}

La visite à domicile permet aux animateurs d'évaluer si l'enfant peut bénéficier de l'expérience de groupe. Nous évitons d'inscrire des enfants tout de suite après le décès, alors que la situation familiale risque d'être encore chaotique et que la réalité du décès n'a pas encore pris racine. Nous les invitons plutôt à participer à une série de séances ultérieure. Si les enfants d'une même 
famille, à peu près du même âge, ne veulent pas être dans le même groupe, les animateurs peuvent proposer à l'un d'eux de participer à la prochaine série de séances.

Les enfants gravement perturbés, extrêmement agressifs ou hyperactifs qu'on ne peut accueillir dans un groupe se voient proposer d'autres types d'intervention, tout comme les familles dont les problèmes sont attribuables à d'autres causes qu'un décès (ex.: lorsqu'un enfant est pris en charge en raison de problèmes familiaux antérieurs).

\section{Durée du programme}

Certains parents et adolescents ont proposé que les séances ne soient pas limitées à une heure et qu'il y en ait davantage. Les animateurs ont souvent eu l'occasion de discuter de cette proposition. On continue à réfléchir sur la durée et le nombre idéal de séances. Certains enfants et parents ont dit que leur participation à une deuxième série de séances leur avait fait du bien. Un certain nombre de groupes de parents et d'adolescents ont continué de se rencontrer après leur dernière séance. On pourrait y voir là un besoin de suivi de la part des anciens participants au programme.

\section{CONCLUSION}

La thérapie de groupe est une façon par laquelle une personne peut vivre son deuil. Nous espérons que l'intervention de groupe auprès des enfants et des adolescents endeuillés aidera à prévenir les problèmes de santé mentale à long terme susceptibles de se produire dans l'avenir. Les évaluations écrites du taux de satisfaction ont été très positives, tant du côté des parents que des enfants, p. ex.: "J'ai appris que je n'étais pas le seul enfant au monde à avoir perdu son père » (Jason, 9 ans) ; «Peu importe ce que tu dis ou penses, ce n'est pas de ta faute que la personne soit morte» (Colin, 8 ans); "J'ai le droit d'être fâchée et de l'exprimer» (Carla, 14 ans); "Je ne me sens plus aussi triste» (Heather, 10 ans). Plusieurs parents indiquent eux aussi qu'ils ont la chance de s'exprimer ouvertement et honnêtement dans une atmosphère positive, "en parlant à des personnes qui écoutent et qui comprennent». "Mes sentiments sont valides»; «Je me sens moins seul»; «J'accepte un peu plus ce qui se passe»; 
«J'ai remarqué un changement chez mes enfants; ils parlent plus ouvertement et partagent leurs expériences avec moi.» Le Programme de soutien aux enfants et aux adolescents endeuillés doit être examiné dans le cadre d'une recherche évaluative. Il est important d'évaluer les résultats à court et à long terme. En cette période économique difficile, cet examen jouera un rôle capital dans la survie du programme.

\section{Références bibliographiques}

AdAMS-Greenly, M. (1987). Helping Children Communicate About Serious lllness and Death (non publié).

BAKER, J.E., M.A. SEDNEY et E. Gross (1992). "Psychological Tasks for Bereaved Children », American Journal of Orthopsychiatry, vol. 62, $\mathrm{n}^{\mathrm{o}} 1$, janvier.

BertoiA, J., et J. AlLAN (1988). "School Management of the Bereaved Child », Elementary School Guidance and Counselling, vol. 23, octobre.

Betz, C.L., et E.C. Poster (1984). «Children's Concepts of Death», Nursing Clinics of North America, vol. 19, n $\mathrm{n}^{\circ} 2$.

DoKA, K. J. (1995). Children Mourning Mourning Children, Hospice Foundation of America.

GRAY, R.E. (1989). «Adolescents' Perceptions of Social Support After the Death of a Parent», Journal of Psychosocial Oncology, vol. 7, $\mathrm{n}^{\circ} 3$.

Grollman, E.A. (1976). Explaining Death to Children, Boston : Beacon Press.

HeegaArd, M. (1988). When Someone Very Special Dies. Children Can Learn to Cope with Grief, Woodland Press.

HEINZER, M. (1995). "Loss of a Parent in Childhood: Attachment and Coping in a Model of Adolescent Resilience », Holistic Nursing Practice, vol. 9, $\mathrm{n}^{\circ} 3$, avril.

HogAN, N.S., et G.B. GREENFIELD (1991). «Adolescent Sibling Bereavement Symptomatology in a Large Community Sample», Journal of Adolescent Research, vol. 6, $\mathrm{n}^{\circ} 1$.

Krementz, J. (1981). How it Feels When a Parent Dies, New York: Knopy.

NATIONAL CHILDHOOD GRIEF INSTITUTE (1991). Endings and Beginnings, Curriculum.

RANDO, T. (1984). Grief, Dying and Death: Clinical Interventions for Caregivers, Research Press.

RAPHAEL, B. (1983). "The Anatomy of Bereavement», dans Basic Books, Chapter 3. The Bereaved Child, New York.

SCHAEFER, D., et C. LyONS (1986). How Do We Tell the Children, New Market Press. 
SEGAL, R.M. (1984). «Helping Children Express Grief Through Symbolic Communication », Social Casework: the Journal of Contemporary Social Work, vol. 65, $\mathrm{n}^{\circ}$ 10, décembre.

TAylor-Brown, J., A. ACHeson et J. FArber (1993). «Kids Can Cope: A Group Intervention for Children Whose Parents Have Cancer », Journal of Psychosocial Oncology, vol. 11, $\mathrm{n}^{\circ} 1$.

Silverman, P., et J.W. WORDEN (1992). "Children's Reactions in the Early Months After the Death of a Parent», American Journal of Orthopsychiatry, vol. 62, $\mathrm{n}^{\circ} 1$, janvier.

Stephenson, J. (1986). Grief of Siblings, Parental Loss of a Child, T.A. Rando (dir.), Research Press Co.

Webb-Boyd, N. (1993). Helping Bereaved Children, A Handbook for Practioners.

Wolfe, Ben (1987). Children Grieve Too: A Four Year Hospital-Based Young Person's Grief Support Program, Duluth, MN : St. Mary's Grief Support Center (non publié).

Worden, W. (1982). Grief Counselling and Grief Therapy, New York: Springer Publ. Co. 
